\title{
Editorial - The First International Technical Conference on Animal Genetic Resources
}

Sustainable management of the world's animal genetic resources is of vital importance to agriculture, food production, rural development and the environment. The first International Technical Conference on Animal Genetic Resources to be held in Interlaken, Switzerland, in September 2007 will raise awareness among stakeholders and promote improved policy development and cooperation at all levels.

The conference will see the launch of The State of the World's Animal Genetic Resources for Food and Agriculture - the first global assessment of the status and management of livestock biodiversity. The State of the World is the culmination of a long process of reporting, analysis, writing and reviewing, which has involved a great number of individuals throughout the world. The 169 Country Reports submitted to FAO were key sources of information, supplemented by reports from international organizations, specially commissioned thematic studies, and the knowledge of the authors and reviewers. The country-based reporting process has already stimulated policy development at national level and led to the elaboration of a Global Plan of Action for Animal Genetic Resources, which once adopted, will provide an agenda for action by the international community. The Interlaken Conference will mark a historic opportunity for the international community to make strategic choices for the future management of animal genetic resources.

This special issue of AGRI reflects the efforts made by governments, INGOs, NGOs, livestock keepers, and FAO in the area of animal genetic resources. It reviews the development of AnGRrelated activities, presents examples of breed improvement and conservation measures, and discusses a number of policy issues. Also included are 17 contributions highlighting national actions in animal genetic resources management that have been implemented subsequent to the preparation of the Country Reports on the State of Animal Genetic Resources. These examples underscore the contribution of the The State of the World process to raising awareness of animal genetic resources, and illustrate that this has led to action at technical and policy levels. They also highlight the need for further action in various aspects of animal genetic resources management.

The adoption of a Global Plan of Action for Animal Genetic Resources is another intended outcome of the Interlaken Conference. The Global Plan of Action will provide a basis, agreed by the international community, to support and increase the overall effectiveness of national, regional and global efforts for the sustainable use, development and conservation of animal genetic resources, and to sustainably mobilize resources. It is intended as a rolling plan with an initial time horizon of ten years. Prospects for the Global Plan of Action are enhanced by a growing awareness that countries are fundamentally interdependent with respect to animal genetic resources for food and agriculture, and that substantial international cooperation is necessary to ensure the effective management of animal genetic diversity.

We gratefully acknowledge the efforts of the authors who contributed articles to this issue and provided their papers at short notice. 


\section{Éditorial - La première Conférence Technique Internationale sur les Ressources Génétiques Animales}

La gestion durables des ressources génétiques animales au niveau mondial est d'une importance vitale pour l'agriculture, la production alimentaire, le développement rural et l'environnement. La première Conférence Technique Internationale sur les Ressources Génétiques Animales qui aura lieu à Interlaken, en Suisse, en septembre 2007, augmentera la sensibilisation entre les parties intéressées du secteur de l'élevage et encouragera l'amélioration des politiques de développement et de la coopération à tous les niveaux.

La conférence servira aussi au lancement du document sur la Situation Mondiale des Ressources Génétiques Animales pour l'Alimentation et l'Agriculture - première évaluation au niveau mondial sur la situation et la gestion de la biodiversité en élevage. La Situation Mondiale est le point culminant d'un long processus fait de rapports, d'analyses, de rédactions et de révisions qui a compté avec un grand nombre d'individus en provenance de différents parties du monde. Les 169 Rapports Nationaux soumis à la FAO ont été une source essentielle d'informations complétées avec les rapports réalisés par les organisations internationales auxquelles avaient été demandé des études sur des thèmes spécifiques, et avec la connaissances des auteurs et des réviseurs. Le processus d'élaboration des rapports nationaux a contribué à la stimulation de politiques de développement au niveau national et a porté à l'élaboration du Plan Mondial d'Action pour les Ressources Génétiques Animales qui, après approbation, permettra d'établir un agenda de travail pour les actions à réaliser par la communauté internationale. La Conférence $\mathrm{d}^{\prime}$ Interlaken offrira une occasion historique à toute la communauté internationale pour définir les choix stratégiques pour la gestion future des ressources génétiques animales.

Ce numéro spécial de AGRI reflète les efforts réalisés par les gouvernements, les INGO et NGO, les éleveurs et la FAO dans le domaine des ressources génétiques animales. Il présente une révision du développement des activités en relation avec les AnGR ainsi que des exemples sur amélioration de race et mesures de conservation, et on discute d'un certain nombre de thèmes politiques. Ce numéro comprend aussi 17 articles qui soulignent les actions au niveau national dans la gestion des ressources génétiques animales qui ont été entreprises comme conséquence de la préparation des Rapports Nationaux sur la Situation des Ressources Génétiques Animales. Ces exemples soulignent aussi l'importance du document sur la Situation Mondiale tout au long du processus de sensibilisation au thème des ressources génétiques animales et illustrent comment il a conduit à des actions concrètes au niveau technique et politique. Le numéro reporte aussi le besoin d'ultérieures actions nécessaires à différents niveaux de la gestion des ressources génétiques animales.

L'adoption du Plan Mondial d'Action pour les Ressources Génétiques Animales est l'autre objectif que nous essayerons d'atteindre pendant la Conférence d'Interlaken. Avec l'accord de la communauté international, le Plan Mondial d'Action donnera les bases nécessaires pour soutenir et augmenter l'efficacité globale au niveaux national, régional et mondial de tous les efforts pour l'utilisation durable, le développement et la conservation des ressources génétiques animales, et la durabilité dans la mobilisation des ressources. Cet évènement est conçu pour établir un objectif initial avec un horizon de dix ans. Les probabilités que le Plan Mondial d'Action soit amélioré grâce à une majeur sensibilisation dépendra si les pays sont fondamentalement interdépendants par rapport aux ressources génétiques animales pour l'alimentation et l'agriculture, et de la coopération internationale qui devra assurer une gestion effective de la diversité génétique animale.

Nous voulons remercier les efforts de tous les auteurs qui ont contribué à ce numéro en envoyant leurs articles avec un si court délai. 


\section{Editorial - La primera Conferencia Técnica Internacional sobre Recursos zoogenéticos}

La gestión sostenible de los recursos zoogenéticos a nivel mundial es de vital importancia para la agricultura, la producción de alimentos, el desarrollo rural y el ambiente. La primera Conferencia Técnica Internacional sobre Recursos Zoogenéticos que tendrá lugar en Interlaken, Suiza, en septiembre 2007, tratará de concienciar la gestión sostenible de los recursos zoogenéticos a nivel mundial es de vital importancia para la agricultura, la producción de alimentos, el desarrollo rural y el ambiente. La primera Conferencia Técnica Internacional sobre Recursos Zoogenéticos que tendrá lugar en Interlaken, Suiza, en septiembre 2007, tratará de concienciar las partes interesadas en el sector ganadero y promoverá la mejora de políticas de desarrollo y la cooperación a todos los niveles.

La conferencia será también el marco para la presentación del documento sobre la Situación Mundial de los Recursos Zoogenéticos para la Alimentación y la Agricultura - primera valoración a nivel mundial sobre la situación y gestión de la biodiversidad ganadera. La Situación Mundial es la culminación de un largo proceso formado de informes, análisis, redacciones y revisiones que ha involucrado un gran número de individuos provenientes de todo el mundo. Los 169 Informes Nacionales sometidos a la FAO han sido una fuente clave de información que ha sido complementada con los informes realizados por las organizaciones internacionales, a las que se han solicitado estudios temáticos específicos, y con el conocimiento de los autores y revisores. El proceso de elaboración de los informes nacionales ha contribuido a estimular las políticas de desarrollo a nivel nacional y ha llevado a la elaboración del Plan Mundial de Acción para los Recursos Zoogenéticos que una vez aprobado permitirá elaborar una agenda de trabajo para las acciones a realizar por parte de la comunidad internacional. La Conferencia de Interlaken proporcionará una oportunidad histórica a toda la comunidad internacional para definir elecciones estratégicas para la futura gestión de los recursos zoogenéticos.

Este número especial de AGRI refleja los esfuerzos realizados por los gobiernos, las INGO y $\mathrm{NGO}$, los ganaderos y la FAO en el terreno de los recursos zoogenéticos. Se hace una revisión del desarrollo de las actividades relacionadas con AnGR, se presentan ejemplos de mejora de raza y medidas de conservación, y se discuten un cierto número de temas políticos. Este número también incluye 17 artículos que subrayan las acciones a nivel nacional en la gestión de los recursos zoogenéticos que han sido realizadas como consecuencia a la preparación de los Informes Nacionales sobre la Situación de los Recursos Zoogenéticos. Estos ejemplos recalcan la importancia del documento sobre la Situación Mundial en todo el proceso de concienciación sobre los recursos zoogenéticos e ilustran cómo ha llevado también a acciones concretas a nivel técnico y político. También se recalca la necesidad de ulteriores acciones necesarias en distintos aspectos de la gestión de los recursos zoogenéticos.

La adopción del Plan Mundial de Acción para los Recursos Zoogenéticos es otro de los logros que se intenta obtener durante la Conferencia de Interlaken. El Plan Mundial de Acción proporcionará las bases, con el acuerdo de la comunidad internacional, para apoyar e incrementar la eficacia global a nivel nacional, regional y mundial de los esfuerzos para la utilización sostenible, el desarrollo y conservación de los recursos zoogenéticos, y la sostenibilidad en la movilización de los recursos. Este evento está concebido para establecer un objetivo inicial con un horizonte de diez años. Las probabilidades de que el Plan Mundial de Acción se vea realzado con una mayor concienciación dependerá de que los países sean fundamentalmente interdependientes con respecto a los recursos zoogenéticos para la alimentación y la agricultura, para lo que será necesaria una cooperación internacional importante para asegurar la gestión efectiva de la diversidad genética animal.

Queremos agradecer los esfuerzos de todos los autores que han contribuido a este número enviando sus artículos con tan previo aviso. 